\title{
Involvement of Sensorimotor, Limbic, and Associative Basal Ganglia Domains in L-3,4-Dihydroxyphenylalanine- Induced Dyskinesia
}

\author{
Céline Guigoni, ${ }^{1}$ Qin Li, ${ }^{3}$ Incarnation Aubert, ${ }^{2}$ Sandra Dovero, ${ }^{1}$ Bernard H. Bioulac, ${ }^{1}$ Bertrand Bloch, ${ }^{2}$ \\ Alan R. Crossman, ${ }^{4}$ Christian E. Gross, ${ }^{1}$ and Erwan Bezard ${ }^{1}$ \\ ${ }^{1}$ Basal Gang, Centre National de la Recherche Scientifique (CNRS) Unité Mixte de Recherche (UMR) 5543, and ²CNRS UMR 5541, Université Victor Segalen- \\ Bordeaux 2, 33076 Bordeaux Cedex, France, ${ }^{3}$ Laboratory Animal Research Center, China Agricultural University, Beijing 100101, China, and ${ }^{4}$ School of \\ Biological Sciences, University of Manchester, Manchester M13 9PT, United Kingdom
}

Dyskinesia represents a debilitating complication of L-3,4-dihydroxyphenylalanine (L-dopa) therapy for Parkinson's disease. Such motor manifestations are attributed to pathological activity in the motor parts of basal ganglia. However, because consistent funneling of information takes place between the sensorimotor, limbic, and associative basal ganglia domains, we hypothesized that nonmotor domains play a role in these manifestations. Here we report the changes in 2-deoxyglucose (2-DG) accumulation in the sensorimotor, limbic, and associative domains of basal ganglia and thalamic nuclei of four groups of nonhuman primates: normal, parkinsonian, parkinsonian chronically treated with L-dopa without exhibiting dyskinesia, and parkinsonian chronically treated with L-dopa and exhibiting overt dyskinesia. Although nondyskinetic animals display a rather normalized metabolic activity, dyskinetic animals are distinguished by significant changes in 2-DG accumulation in limbic- and associative-related structures and not simply in sensorimotorrelated ones, suggesting that dyskinesia is linked to a pathological processing of limbic and cognitive information. We propose that these metabolic changes reflect the underlying neural mechanisms of not simply motor dyskinesias but also affective, motivational, and cognitive disorders associated with long-term exposure to L-dopa.

Key words: 2-deoxyglucose; globus pallidus pars externalis; subthalamic nucleus; mediodorsal nucleus; nucleus stria terminalis; basal ganglia

\section{Introduction}

Chronic treatment of Parkinson's disease $(\mathrm{PD})$ patients with the dopamine precursor L-3,4-dihydroxyphenylalanine (L-dopa) induces development of adverse fluctuations in motor response and involuntary movements, known as L-dopa-induced dyskinesia (LID) (Yahr et al., 1968). The motor nature of these manifestations led to investigation of the abnormalities of neuronal function in the cortico-basal ganglia-thalamocortical motor circuit (for review, see Bezard et al., 2001b). Although a current theory concerning the organization of basal ganglia pathways proposes that the frontal cortex and the basal ganglia are arranged in parallel, functionally segregated circuits (Alexander and Crutcher, 1990), there is supporting evidence for consistent funneling of

\footnotetext{
Received July 20, 2004; revised Jan. 12, 2005; accepted Jan. 15, 2005

This work was supported by grants from the Michael J. Fox Foundation for Parkinson's Research (E.B., B.B.), the Fédération pour la Recherche sur le Cerveau (E.B., C.E.G.), and the Fondation pour la Recherche Médicale (E.B. C.E.G.). C.G. is a postdoctoral fellow funded through a grant from the Michael J. Fox Foundation for Parkinson's Research. The University Victor Segalen, the Centre National de la Recherche Scientifique (CNRS), and the Institut Fédératif de Recherches of Neuroscience (Institut National de la Santé et de la Recherche Médicale Number 8; CNRS Number 13) provided the infrastructural support. We thank L. Cardoit for technical assistance.

Correspondence should be addressed to Erwan Bezard, Basal Gang, Laboratoire de Physiologie et Physiopathologie de la Signalisation Cellulaire, Centre National de la Recherche Scientifique Unité Mixte de Recherche 5543, Université Victor Segalen, 146 rue Léo Saignat, 33076 Bordeaux Cedex, France. E-mail: erwan.bezard@umr5543.u-bordeaux2.fr. DOI:10.1523/JNEUROSC1.5059-04.2005

Copyright $\odot 2005$ Society for Neuroscience $\quad 0270-6474 / 05 / 252102-06 \$ 15.00 / 0$
}

information between the so-called sensorimotor, limbic, and associative domains (Nauta and Domesick, 1978; Haber et al., 1993, 2000).

Interestingly, Mitchell et al. showed in their 2-deoxyglucose (2-DG) seminal studies (Mitchell et al., 1989, 1992) that the motor component of the subthalamic nucleus (STN), the dorsolateral tip, had normal terminal activity in chorea that was increased significantly in dystonia, whereas the metabolic activity of the ventromedial "limbic/associative" STN was impaired in both cases. This result originally suggested pathophysiological differences between L-dopa-induced chorea and dystonia. We now hypothesize that it may also reflect the involvement of nonmotor pathways in LID, raising the possibility that LID also corresponds to an abnormal processing of limbic/associative information.

In this study, we report the changes in 2-DG accumulation (Sokoloff et al., 1977) in the sensorimotor, limbic, and associative domains of basal ganglia and thalamic nuclei of four nonhuman primate groups: normal, parkinsonian, parkinsonian chronically treated with L-dopa without exhibiting dyskinesia, and parkinsonian chronically treated with L-dopa exhibiting overt dyskinesia.

\section{Materials and Methods}

Animals. Nineteen female Macaca fascicularis monkeys (Shared Animal Health, Beijing, People's Republic of China) were housed in individual 


\begin{tabular}{|c|c|c|c|c|c|c|c|c|c|c|c|}
\hline \multirow[b]{3}{*}{ Groups } & \multirow{3}{*}{$\begin{array}{l}\text { DAT binding } \\
\text { (fmol/mg) }\end{array}$} & \multicolumn{3}{|c|}{ TH and NissI cells } & \multicolumn{7}{|c|}{ L-Dopa administration } \\
\hline & & \multirow[b]{2}{*}{ TH } & \multirow[b]{2}{*}{ NS } & \multirow[b]{2}{*}{ TH + NS } & \multirow[b]{2}{*}{ Dose (mg) } & \multicolumn{2}{|c|}{ Scores at $0 \mathrm{~min}$} & \multicolumn{2}{|c|}{ Scores at $30 \mathrm{~min}$} & \multicolumn{2}{|c|}{ Scores at $60 \mathrm{~min}$} \\
\hline & & & & & & $P$ & $D$ & $P$ & $D$ & $P$ & $D$ \\
\hline $\mathrm{C} 1$ & 123.9 & 922 & 139 & 1061 & & 0.0 & 0.0 & & & & \\
\hline $\mathrm{C} 2$ & 154.5 & 958 & 113 & 1071 & & 0.0 & 0.0 & & & & \\
\hline $\mathrm{C} 3$ & 134.9 & 1007 & 107 & 1114 & & 0.0 & 0.0 & & & & \\
\hline \multicolumn{12}{|l|}{ MPTP } \\
\hline M1 & 1.6 & 137 & 216 & 353 & & 8.0 & 0.0 & & & & \\
\hline M2 & 3.4 & 140 & 290 & 430 & & 9.0 & 0.0 & & & & \\
\hline M3 & 2.7 & 74 & 237 & 311 & & 7.5 & 0.0 & & & & \\
\hline M4 & 3.5 & 198 & 211 & 409 & & 9.5 & 0.0 & & & & \\
\hline M5 & 1.5 & 135 & 244 & 379 & & 8.5 & 0.0 & & & & \\
\hline L4 & 5.3 & 134 & 270 & 404 & 50 & 8.4 & 0.0 & 2.2 & 0.0 & 0.0 & 0.4 \\
\hline \multicolumn{12}{|c|}{ MPTP/L-dopa dyskinetic } \\
\hline D1 & 8.6 & 246 & 234 & 480 & 60 & 8.8 & 0.0 & 2.0 & 0.0 & 0.7 & 3.1 \\
\hline $\mathrm{D} 2$ & 5.6 & 217 & 217 & 434 & 66 & 7.5 & 0.0 & 1.9 & 0.0 & 0.3 & 2.7 \\
\hline D3 & 7.7 & 106 & 164 & 270 & 53 & 7.2 & 0.0 & 1.8 & 0.0 & 0.0 & 3.1 \\
\hline D4 & 0.8 & 155 & 213 & 369 & 50 & 7.5 & 0.0 & 2.8 & 0.0 & 0.0 & 2.9 \\
\hline D5 & 4.1 & 178 & 237 & 415 & 55 & 10.2 & 0.0 & 2.1 & 0.0 & 0.2 & 2.8 \\
\hline
\end{tabular}

Mean age, $3.05 \pm 0.05$ years; mean weight, $3.1 \pm 0.1 \mathrm{~kg}$. Extent of lesion is comparable among the three MPTP-lesioned groups both at the striatal [dopamine transporter (DAT) binding; femtomoles per milligram of equivalent tissue] and nigral [tyrosine hydroxylase (TH)-immunopositive neurons and Nissl-stained (NS) cells] levels [for methods, see Bezard et al. (2001c, 2003)]. L-Dopa dose (in milligrams) is given for each individual as well as their mean parkinsonian (P) and mean dyskinetic (D) scores before administration ( $0 \mathrm{~min}$ ) and at 30 and $60 \mathrm{~min}$ after administration. The only dyskinetic animals displayed severe dyskinesia 60 min after $\mathrm{L}-$ dopa administration (Bezard et al., 2003 ), presenting choreic-athetoid (characterized by constant writhing and jerking motions), dystonic, and sometimes ballistic movements (large-amplitude flinging, flailing movements). At the peak of the dose (60 - 150 min after injection), dystonic rolling and writhing on the cage floor were common.

primate cages under controlled conditions of humidity (50 $\pm 5 \%$ ), temperature $\left(24 \pm 1^{\circ} \mathrm{C}\right)$, and light $(12 \mathrm{~h}$ light/dark cycles $)$; food and water were available ad libitum, and animal care was supervised by veterinarians. Experiments were performed in accordance with the European Communities Council Directive of November 24, 1986 (86/609/EEC) for care of laboratory animals.

Experimental protocol. Population characteristics (summarized in Table 1) indicate that all 1-methyl-4-phenyl-1,2,3,6-tetrahydropyridine (MPTP)-treated groups showed a comparable level of lesion but different behaviors (Bezard et al., 2001c, 2003). Control and MPTP-treated animals correspond to the animals named D0 and D25 groups in the following studies (Bezard et al., 2001a,c), whereas nondyskinetic and dyskinetic animals come from a population of animals validated previously (Bezard et al., 2003). Those later were chronically treated with twice daily administration of L-dopa (Modopar; Roche, Welwyn Garden City, UK; L-dopa/ carbidopa ratio, 4:1) for 6-8 months at a tailored dose designed to produce a full reversal of the parkinsonian condition ( $16-20 \mathrm{mg} / \mathrm{kg} \mathrm{L}-\mathrm{dopa})$. Five monkeys developed severe and reproducible dyskinesia (MPTPintoxicated, dyskinetic monkey group), whereas four did not (MPTPintoxicated, nondyskinetic monkey group).

Behavioral assessment. The parkinsonian condition was assessed on a parkinsonian monkey rating scale using videotape recordings of monkeys (Boraud et al., 2001; Bezard et al., 2003). A score of 0 corresponds to a normal animal, and a score of $>6$ corresponds to a parkinsonian animal (Bezard et al., 2003). The severity of dyskinesia was rated using the dyskinesia disability scale (Pearce et al., 1995; Brotchie and Fox, 1999): 0, dyskinesia absent; 1 , mild, fleeting, and rare dyskinetic postures and movements; 2 , moderate, more prominent abnormal movements, but not interfering significantly with normal behavior; 3, marked, frequent, and, at times, continuous dyskinesia intruding on the normal repertoire of activity; or, 4 , severe, virtually continuous dyskinetic activity, disabling to the animal and replacing normal behavior.

2-DG procedure. On the day they were killed, animals were given an intravenous injection of $1 \mathrm{mCi} / \mathrm{kg}\left[{ }^{3} \mathrm{H}\right] 2-\mathrm{DG}$ (specific activity, $50 \mathrm{Ci} /$ mmol, $185 \mathrm{GBq} / \mathrm{mmol}$; Interchim, Grenoble, France) in sterile saline as described previously (Mitchell et al., 1989; Bezard et al., 2001a). After 45 $\mathrm{min}$, all animals were killed by sodium pentobarbital overdose $(150 \mathrm{mg} /$ $\mathrm{kg}$, i.v.). L-Dopa-treated animals received their tailored dose of L-dopa 15 min before 2-DG (Table 1). However, a single individual, D5, received its L-dopa 15 min after 2-DG (Table 1). Brains were quickly removed, immediately frozen in isopentane $\left(-45^{\circ} \mathrm{C}\right)$, and stored at $-80^{\circ} \mathrm{C}$. Tissue was sectioned at $20 \mu \mathrm{m}$ in a cryostat at $-17^{\circ} \mathrm{C}$ and thaw-mounted onto gelatin-subbed slides. Once freeze-dried $\left(-60^{\circ} \mathrm{C} ; 40.10^{-3}\right.$ atmospheres) for $2 \mathrm{~h}$, both serial sections and autoradiographic methylmethacrylate standards (Amersham Biosciences, Uppsala, Sweden) were exposed to ${ }^{3} \mathrm{H}$-Hyperfilm (Amersham Biosciences) for 2 months at $-30^{\circ} \mathrm{C}$, developed in D-19 developer (Eastman Kodak, Rochester, NY), and fixed in Kodak Unifix. Densitometric analysis of autoradiographs was performed using an image analysis system (Visioscan version 4.12; Biocom, Les Ulis, France) as described previously (Bezard et al., 2001a). Four sections per animal per nucleus were analyzed by an examiner blind with regard to the experimental condition. Optical densities were averaged for each region in each animal and converted to the amount of radioactivity bound in comparison with the standards. Mean bound radioactivity and SEM were then calculated for each group.

Statistical analysis. For multiple comparisons of parametric data, oneway ANOVA was used to estimate overall significance, followed by post hoc $t$ tests corrected for multiple comparisons by Bonferroni's method (Miller, 1981). For multiple comparisons of behavioral assessments, a Kruskal-Wallis nonparametric test was used to estimate overall significance, followed by post hoc $t$ tests corrected for multiple comparisons by Dunn's method (Miller, 1981). All data were normally distributed, and significance levels of $t$ test comparisons were adjusted for inequality of variances when appropriate. These analyses were completed using the Stata program (Intercooled Stata 6.0; Stata Corporation, College Station, TX). $p<0.05$ was considered significant. 


\section{Results}

2-DG uptake was measured across sensorimotor, associative, and limbic domains of basal ganglia and in some target structures of these three domains. For that purpose, the external part of the globus pallidus (GPe) was divided into sensorimotor (GPe-M), associative (GPe-A) and limbic (GPe-L) territories, as defined previously (Haber et al., 1993; Francois et al., 1994) (Fig. 1). The STN was divided into sensorimotor (STN-M) and associative-limbic (STN-AL) domains (Parent and Hazrati, 1995b) (Fig. 1). 2-DG accumulation was measured as a whole in the internal part of the globus pallidus (GPi) and in the substantia nigra pars reticulata $(\mathrm{SNr})$, two output structures of basal ganglia, because of the consistent overlap between different domains in these nuclei (Parent and Hazrati, 1995a). Three nuclei were studied in the thalamus. The ventral anterior (VA) and ventral lateral (VL) nuclei are defined as thalamic targets of the motor loop. VA nuclei were measured on sections $4 \mathrm{~mm}$ anterior to anterior commissura (AC) where the STN is absent, and VL nuclei were measured on sections $6 \mathrm{~mm}$ to AC where the STN is present (Martin and Bowden, 1996). The mediodorsal nucleus (MD) is defined as thalamic output of the associative loop of the basal ganglia (Parent and Hazrati, 1995a). The nucleus of the stria terminalis (NStT) was chosen as a limbic output of basal ganglia (Groenewegen et al., 1991).

In the three domains of the GPe (GPe-M, $F_{(3,17)}=7.01, p<$ 0.005 ; GPe-A, $F_{(3,17)}=14.51, p<0.0001 ; \mathrm{GPe}-\mathrm{L}, F_{(3,17)}=11.33$, $p<0.0005), 2-D G$ uptake was significantly elevated in parkinsonian animals compared with normal animals $(p<0.05)$ (Fig. 1 , Table 2). Both the nondyskinetic and dyskinetic animals displayed apparently normalized levels of 2-DG accumulation in the GPe-M and GPe-L (Fig. 1, Table 2). However, dyskinetic animals showed significantly decreased 2-DG uptake in the GPe-A compared with control, MPTP-treated, and nondyskinetic animals $(p<0.05)$ (Fig. 1, Table 2).

2-DG uptake in the two domains defined in the STN was modified as well (STN-M, $F_{(3,17)}=17.51, p<0.0001$; STN-AL, $\left.F_{(3,17)}=29.56, p<0.0001\right)$. The MPTP-treated animals showed a significant decrease in 2-DG accumulation in both the STN-M and STN-AL $(p<0.05)$ (Fig. 1, Table 2). In the STN-M, L-dopa treatment induced an increase in 2-DG levels in both the nondyskinetic and the dyskinetic group; no significant difference was observed with the control group. Surprisingly, in the STN-AL, this increased 2-DG uptake was more marked. Both nondyskinetic and dyskinetic animals showed a significant increase in 2-DG accumulation compared with MPTP but also with normal animals $(p<0.05)$ (Fig. 1, Table 2).

In the GPi $\left(F_{(3,17)}=16.32 ; p<0.0001\right)$, MPTP treatment induced a significant increase in 2-DG uptake compared with control animals $(p<0.05)$ (Fig. 1, Table 2). L-Dopa administration did not induce changes in 2-DG uptake in the nondyskinetic L-dopa-treated, MPTP-treated group (Fig. 1, Table 2). On the contrary, in the dyskinetic group, the 2-DG accumulation was higher than in the MPTP-treated group $(p<0.05)$ (Fig. 1, Table 2 ). No significant change was observed in 2-DG uptake in the $\mathrm{SNr}$
$\left(F_{(3,17)}=3.61 ; p<0.05\right)$ in any group, as reported previously (Mitchell et al., 1989, 1992; Bezard et al., 2001a).

In both VA nuclei $\left(F_{(3,17)}=12.04 ; p<0.0004\right)$ and VL nuclei $\left(F_{(3,17)}=14.50 ; p<0.0001\right)$, the motor nuclei of the thalamus, the MPTP treatment induced a strong and significant increase in 2 -DG uptake $(p<0.05)$ (Fig. 1, Table 2). In the nondyskinetic and dyskinetic groups, 2-DG uptake is reduced by L-dopa therapy in both VA and VL nuclei, leading to an apparent normalization of 2-DG levels compared with control animals (Fig. 1, Table 2). Such normalization makes 2-DG uptake in VA and VL nuclei significantly different from the parkinsonian situation (Fig. 1, Table 2). In the $\operatorname{MD}\left(F_{(3,17)}=6.42 ; p<0.0058\right)$, an associative nucleus, no change was observed in either the MPTP-treated or the nondyskinetic groups. However, the dyskinetic animals showed significantly decreased 2-DG uptake compared with the other three groups $(p<0.05)$ (Fig. 1, Table 2).

In the $\operatorname{NStT}\left(F_{(3,17)}=3.52 ; p<0.05\right), 2-D G$ uptake was modified only in the dyskinetic animals. In this group, the level of 2-DG was significantly reduced compared with the control group $(p<0.05)$ (Fig. 1, Table 2).

The above results suggested that, whereas nondyskinetic animals displayed normalized metabolic activity compared with controls, dyskinetic animals were distinguished by significant changes in 2-DG accumulation in limbic- and associative-related structures and not simply in sensorimotor nuclei. These results were obtained using all animals but D5 that had been killed 30 min after L-dopa administration (see Materials and Methods). At this time point, D5 parkinsonian symptoms were improved, but no LID was rated (Table 1). Indeed, LID appeared from 45 to 50 min onward in the dyskinetic group (Bezard et al., 2003). Although data deriving from a single individual cannot be used for statistical analysis, the qualitative analysis provides interesting insights (Fig. 1, Table 2). At $30 \mathrm{~min}$ after L-dopa administration, 2-DG levels in motor nuclei (e.g., in the GPe-M, STN-M, VA nuclei, and VL nuclei) were not different (defined as within 1 SEM) from both nondyskinetic and dyskinetic animals at $60 \mathrm{~min}$ after L-dopa administration, in direct correlation with improvement of parkinsonian motor abnormalities (Table 1). In the as- 
Table 2. Densitometric analysis of 2-DG accumulation in control, parkinsonian (MPTP), L-dopa nondyskinetic, and L-dopa dyskinetic animals

\begin{tabular}{|c|c|c|c|c|c|c|c|c|c|}
\hline \multirow[b]{3}{*}{ Area } & \multirow[b]{3}{*}{ Control (mean \pm SEM) } & \multirow{2}{*}{\multicolumn{2}{|c|}{ MPTP }} & \multirow{2}{*}{\multicolumn{2}{|c|}{ L-Dopa nondyskinetic }} & \multicolumn{4}{|c|}{ L-Dopa dyskinetic } \\
\hline & & & & & & \multicolumn{2}{|l|}{$30 \mathrm{~min}$} & \multicolumn{2}{|l|}{$60 \mathrm{~min}$} \\
\hline & & Mean \pm SEM & $\%$ & Mean \pm SEM & $\%$ & Mean & $\%$ & Mean \pm SEM & $\%$ \\
\hline GPe-M & $1.68 \pm 0.11$ & $1.96 \pm 0.10^{*}$ & 16.67 & $1.75 \pm 0.16$ & 4.17 & 1.71 & 1.78 & $1.66 \pm 0.09$ & -1.19 \\
\hline GPe-A & $1.65 \pm 0.10$ & $1.87 \pm 0.11^{*}$ & 13.34 & $1.67 \pm 0.08^{\dagger}$ & 1.21 & 1.71 & 3.63 & $1.45 \pm 0.08^{* \dagger \neq}$ & -12.12 \\
\hline GPe-L & $1.67 \pm 0.11$ & $1.95 \pm 0.12^{*}$ & 16.77 & $1.79 \pm 0.12$ & 7.19 & 1.72 & 2.99 & $1.50 \pm 0.13^{\dagger \ddagger}$ & -10.18 \\
\hline STN-M & $2.22 \pm 0.14$ & $1.83 \pm 0.18^{*}$ & -17.57 & $2.24 \pm 0.11^{\dagger}$ & 0.90 & 2.33 & 4.95 & $2.51 \pm 0.11 \dagger$ & 13.06 \\
\hline STN-AL & $2.52 \pm 0.19$ & $2.10 \pm 0.15^{*}$ & -16.67 & $2.85 \pm 0.12^{* \dagger}$ & 13.09 & 2.67 & 5.95 & $2.91 \pm 0.09^{* \dagger}$ & 15.48 \\
\hline GPi & $1.81 \pm 0.14$ & $2.14 \pm 0.21^{*}$ & 18.23 & $2.24 \pm 0.12^{*}$ & 23.76 & 2.22 & 22.65 & $2.51 \pm 0.11^{* \dagger}$ & 38.67 \\
\hline SNr & $1.93 \pm 0.18$ & $2.15 \pm 0.16$ & 11.39 & $1.93 \pm 0.16$ & 0 & 1.97 & 2.07 & $2.24 \pm 0.19$ & 16.06 \\
\hline VA & $1.82 \pm 0.15$ & $2.13 \pm 0.08^{*}$ & 17.03 & $1.76 \pm 0.07$ & -3.29 & 1.71 & -6.04 & $1.67 \pm 0.17$ & -8.24 \\
\hline$V L$ & $1.93 \pm 0.16$ & $2.40 \pm 0.16^{*}$ & 24.35 & $1.97 \pm 0.12$ & 2.07 & 1.85 & -4.1 & $1.80 \pm 0.13$ & -6.74 \\
\hline MD & $1.71 \pm 0.07$ & $1.79 \pm 0.08$ & 4.68 & $1.73 \pm 0.17$ & 1.17 & 1.69 & -1.17 & $1.48 \pm 0.11^{* \dagger \neq}$ & -13.45 \\
\hline NStT & $1.87 \pm 0.08$ & $1.83 \pm 0.12$ & -2.14 & $1.77 \pm 0.15$ & -5.35 & 1.81 & -3.21 & $1.64 \pm 0.10^{*}$ & -12.29 \\
\hline
\end{tabular}

Data (mean \pm SEM) are expressed in terms of tissue equivalent ratios of the amount of radioactivity in the considered structure to that in the white matter of the same section. \% represents the mean difference with controls. Because a single individual is used 30 min after L-dopa administration, no SEM is given and no statistical analysis has been performed. ${ }^{*} p<0.05$, significant difference with control; ${ }^{\dagger} p<0.05$, significant difference with MPTP; ${ }^{\ddagger} p<0.05$, significant difference with nondyskinetic.

sociative-limbic nuclei, however, 2-DG levels were similar to nondyskinetic animals and different (defined as beyond 1 SEM) from the dyskinetic animals, although D5 displayed the same temporal pattern for LID appearance as the four other dyskinetic animals (i.e., LID would occur 15-20 min later) (Table 2).

\section{Discussion}

The key finding of this study is that the neural mechanisms responsible for LID expression may also involve both limbic and associative domains and not simply the sensorimotor domain. Qualitative temporal analysis also supports this view, because in a dyskinetic animal killed when parkinsonian motor abnormalities were improved but before LID appeared, 2-DG levels were normalized in motor nuclei and not different from nondyskinetic animals in associative-limbic nuclei. We propose, therefore, that dyskinesia is linked to pathological metabolic activity in the associative-limbic nuclei. LID should not be regarded simply as a movement disorder but also as involving affective, motivational, and cognitive aspects of behavior, which have thus far been poorly investigated in the clinic.

The pattern of changes in 2-DG accumulation in our experimental groups matches the original findings of Mitchell et al. $(1989,1992)$. For instance, we do confirm the differential metabolic activity between the motor component of the STN, the dorsolateral tip, and the ventromedial limbic-associative STN in both nondyskinetic and dyskinetic animals. Although they showed normalized metabolic activity in the GPe of dyskinetic animals compared with normal animals, we show that this occurs only in the sensorimotor domain but not in both the limbic and associative domains, where the uptake is significantly decreased (Fig. 1). Surprisingly, the metabolic activity of basal ganglia motor-related structures was not affected in the dyskinetic animals compared with the nondyskinetic ones. Increased 2-DG uptake in the GPi should correspond to the increased GABAergic tone coming from the striatum but also from the GPe. Although 2-DG uptake is not changed in the sensorimotor GPe of dyskinetic animals, both the associative and limbic domains showed decreased accumulation, suggestive of a decreased inhibition from the striatum. Interestingly, D5, a dyskinetic animal, did not show such decreased levels in GPe-A/GPe-L domains. Because D5 had been killed before LID appeared, decreased inhibition from the associative-limbic striatum would not occur when parkinsonian motor abnormalities were improved and when LID was not yet present. Such disinhibition of the GPe should trans- late into increased GABAergic tone from the GPe on the GPi. For the three functional domains overlapping within the GPi, the increased 2-DG accumulation in the GPi would reflect the increased activity arising from the associative-limbic domains of GPe and not from the motor domain. Such an increased GABAergic tone on the GPi is likely responsible for the excessively decreased firing frequency of GPi neurons during LID (Bezard et al., 2001b; Boraud et al., 2001). Both VA and VL nuclei receive extensive GABAergic projections from the GPi; the decreased 2-DG accumulation in those structures in dyskinetic animals was anticipated as reported previously (Mitchell et al., 1992).

Accompanying the decreased 2-DG uptake in the limbic and associative domains of the GPe is the finding that both the NStT and the MD display decreased 2-DG accumulation (1) only in the dyskinetic animals and (2) only when LID is actually present (i.e., not in D5, which was killed before the appearance of LID) (Fig. 1, Table 2). The NStT is a direct output of the ventral striatum, part of the limbic system. A decreased uptake in the NStT is thus suggestive of a decreased GABAergic tone arising from the ventral striatum in dyskinetic animals and only in this later group. Interestingly, an excitatory pathway has been described between the NStT and the STN (F. Georges, personal communication) providing an alternative way to drive the pathological activity of the STN toward LID. In the limbic circuit, the ventral striatum receives projections, notably from the anterior cingulate, area and projects to the ventral commissural GPe, also called the GPe-L (Alexander and Crutcher, 1990; Parent and Hazrati, 1995a). In the associative/cognitive circuit, the rostral striatum receives projections from the associative cortices and projects to the commissural GPe-A. The GPe-A and GPe-L project to the STN-AL, which in turn projects to the GPi (Alexander and Crutcher, 1990; Parent and Hazrati, 1995a). The associative-limbic circuit is closed by GPi projections to the MD, the thalamic target of basal ganglia associative-limbic output. The decreased 2-DG accumulation in the MD of dyskinetic animals would thus be the signature of the specific impairment of the entire circuit. However, although the present study shows a concomitant change, it does not establish a causative link that remains to be directly demonstrated.

Although basal ganglia pathways are thought to be arranged in parallel, functionally segregated circuits (Alexander and Crutcher, 1990), there is supporting evidence for consistent fun- 
neling of information between the so-called sensorimotor, limbic, and associative domains (Nauta and Domesick, 1978; Mogenson et al., 1980; Haber et al., 1993, 2000). Haber et al. (2000) have further developed this concept by proposing that the same information is progressively processed by the different functional domains of the basal ganglia through specific pathways of functional interaction through basal ganglia circuits (Haber et al., 2000; McFarland and Haber, 2002). This hypothesis has recently received support from the demonstration that cocaine selfadministration produces a progressive involvement of limbic, associative, and ultimately sensorimotor striatal domains (Porrino et al., 2004). Both chronic cocaine administration and chronic L-dopa treatment result in elevated dopaminergic tone in the striatum. A nonspecific increase in extracellular dopamine levels within the striatum would lead to widespread dissemination of information instead of focused processing. Here we provide evidence for comparable mechanisms, although not in the same order (sensorimotor, then limbic and associative domains), in LID. Supporting this notion are the investigations of the compartmental changes in expression of FosB proteins in the intact and dopamine-depleted striatum after chronic apomorphine treatment in the rat (Saka et al., 1999; Cenci, 2002). Chronic apomorphine treatment led to increased ventromedial expression of FosB (Saka et al., 1999). Cenci (2002) also demonstrated that such increased FosB immunoreactivity could be related to locomotive motor abnormalities. In other words, hyperdopaminergic tone would lead to pathological involvement of all basal ganglia domains in the processing of previously segregated information.

Along with clinical observations reporting reward-deficiency syndrome or learning deficits in L-dopa-treated PD patients (Swainson et al., 2000; Czernecki et al., 2002), we propose that dyskinesia should not be regarded simply as a movement disorder but also as an affective, motivational, and cognitive disorder. Although the qualitative temporal analysis suggests a causative role for involvement of associative and limbic nuclei in inducing LID, direct electrophysiological measurements are mandatory to formally establish such a causal relationship. Indeed, a change in 2-DG uptake does not necessarily indicate an alteration in the firing activity of neurons but reflects the integration of changes during $45 \mathrm{~min}$ in the overall level of intrinsic synaptic activity (Schwartz et al., 1979). Nonetheless, a fundamental consequence of the present study is that we cannot continue analyzing LID by investigating only motor areas, thus rendering unreliable all studies that do not pay attention to the anatomofunctional organization of cortico-basal ganglia loops. Seeing LID as either "caused" by unwanted involvement of associative and limbic areas or simply as having the cognitive and limbic abnormal counterparts of LID ("consequence"), as is often reported in hyperkinetic disorders (Klawans, 1988; Litvan et al., 1998), might have different clinical consequences. For instance, if electrophysiological investigations support the causative hypothesis, modulating the activity of nonmotor regions would reduce severity of LID, thereby offering new drug targets for treatment of this debilitating condition.

\section{References}

Alexander GE, Crutcher MD (1990) Functional architecture of basal ganglia circuits: neural substrates of parallel processing. Trends Neurosci 13:266-271.

Bezard E, Crossman AR, Gross CE, Brotchie JM (2001a) Structures outside the basal ganglia may compensate for dopamine loss in the presymptomatic stages of Parkinson's disease. FASEB J 15:1092-1094.

Bezard E, Brotchie JM, Gross CE (2001b) Pathophysiology of levodopa- induced dyskinesia: potential for new therapies. Nat Rev Neurosci 2:577-588.

Bezard E, Dovero S, Prunier C, Ravenscroft P, Chalon S, Guilloteau D, Bioulac B, Brotchie JM, Gross CE (2001c) Relationship between the appearance of symptoms and the level of nigrostriatal degeneration in a progressive MPTP-lesioned macaque model of Parkinson's disease. J Neurosci 21:6853-6861.

Bezard E, Ferry S, Mach U, Stark H, Leriche L, Boraud T, Gross CE, Sokoloff P (2003) Attenuation of levodopa-induced dyskinesia by normalizing dopamine D3 receptor function. Nat Med 9:762-767.

Boraud T, Bezard E, Bioulac B, Gross C (2001) Dopamine agonist-induced dyskinesias are correlated to both firing pattern and frequency alteration of pallidal neurons in the MPTP-treated monkey. Brain 124:546-557.

Brotchie JM, Fox SH (1999) Quantitative assessment of dyskinesias in subhuman primates. Mov Disord 14 [Suppl 1]:40-47.

Cenci MA (2002) Transcription factors involved in the pathogenesis of L-DOPA-induced dyskinesia in a rat model of Parkinson's disease. Amino Acids 23:105-109.

Czernecki V, Pillon B, Houeto JL, Pochon JB, Levy R, Dubois B (2002) Motivation, reward, and Parkinson's disease: influence of dopatherapy. Neuropsychologia 40:2257-2267.

Francois C, Yelnik J, Percheron G, Fenelon G (1994) Topographic distribution of the axonal endings from the sensorimotor and associative striatum in the macaque pallidum and substantia nigra. Exp Brain Res 102:305-318.

Groenewegen HJ, Berendse HW, Meredith GE, Haber SN, Voorn P, Wolters JG, Lohman AHM (1991) Functional anatomy of the ventral, limbic system-innervated striatum. In: The mesolimbic dopamine system: from motivation to action (Willner P, Scheel-Krüger J, eds), pp 19-59. New York: Wiley.

Haber SN, Lynd-Balta E, Mitchell SJ (1993) The organization of the descending ventral pallidal projections in the monkey. J Comp Neurol 329:111-128.

Haber SN, Fudge JL, McFarland NR (2000) Striatonigrostriatal pathways in primates form an ascending spiral from the shell to the dorsolateral striatum. J Neurosci 20:2369-2382.

Klawans HL (1988) Psychiatric side effects during the treatment of Parkinson's disease. J Neural Transm Suppl 27:117-122.

Litvan I, Paulsen JS, Mega MS, Cummings JL (1998) Neuropsychiatric assessment of patients with hyperkinetic and hypokinetic movement disorders. Arch Neurol 55:1313-1319.

Martin RF, Bowden DM (1996) A stereotaxic template atlas of the macaque brain for digital imaging and quantitative neuroanatomy. NeuroImage 4:119-150.

McFarland NR, Haber SN (2002) Thalamic relay nuclei of the basal ganglia form both reciprocal and nonreciprocal cortical connections, linking multiple frontal cortical areas. J Neurosci 22:8117-8132.

Miller R (1981) Simultaneous statistical inference. New York: Springer.

Mitchell IJ, Clarke CE, Boyce S, Robertson RG, Peggs D, Sambrook MA, Crossman AR (1989) Neural mechanisms underlying parkinsonian symptoms based upon regional uptake of 2-deoxyglucose in monkeys exposed to 1-methyl-4-phenyl-1,2,3,6-tetrahydropyridine. Neuroscience 32:213-226.

Mitchell IJ, Boyce S, Sambrook MA, Crossman AR (1992) A 2-deoxyglucose study of the effects of dopamine agonists on the parkinsonian primate brain. Implications for the neural mechanisms that mediate dopamine agonist-induced dyskinesia. Brain 115:809-824.

Mogenson GJ, Jones DL, Yim CY (1980) From motivation to action: functional interface between the limbic system and the motor system. Prog Neurobiol 14:69-97.

Nauta WJH, Domesick VB (1978) Crossroads of limbic and striatal circuitry: hypothalamic-nigral connections. In: Limbic mechanisms (Livingston KE, Hornykiewicz O, eds), pp 75-93. New York: Plenum.

Parent A, Hazrati LN (1995a) Functional anatomy of the basal ganglia. I. The cortico-basal ganglia-thalamo-cortical loop. Brain Res Brain Res Rev 20:91-127.

Parent A, Hazrati LN (1995b) Functional anatomy of the basal ganglia. II. The place of subthalamic nucleus and external pallidum in basal ganglia circuitry. Brain Res Brain Res Rev 20:128-154.

Pearce RK, Jackson M, Smith L, Jenner P, Marsden CD (1995) Chronic L-dopa administration induces dyskinesias in the MPTP-treated common marmoset (Callithrix jacchus). Mov Disord 10:731-740. 
Porrino LJ, Lyons D, Smith HR, Daunais JB, Nader MA (2004) Cocaine self-administration produces a progressive involvement of limbic, association, and sensorimotor striatal domains. J Neurosci 24:3554-3562.

Saka E, Elibol B, Erdem S, Dalkara T (1999) Compartmental changes in expression of c-Fos and FosB proteins in intact and dopamine-depleted striatum after chronic apomorphine treatment. Brain Res Brain Res Rev 825:104-114.

Schwartz WJ, Smith CB, Davidsen L, Savaki H, Sokoloff L, Mata M, Fink DJ, Gainer H (1979) Metabolic mapping of functional activity in the hypothalamo-neurohypophysial system of the rat. Science 205: 723-725.

Sokoloff L, Reivich M, Kennedy C, Des Rosiers MH, Patlak CS, Pettigrew KD,
Sakurada O, Shinohara M (1977) The $\left[{ }^{14} \mathrm{C}\right]$-deoxyglucose method for the measurement of local cerebral glucose utilization: theory, procedure, and normal values in the conscious and anesthetized albino rat. J Neurochem 28:897-916.

Swainson R, Rogers RD, Sahakian BJ, Summers BA, Polkey CE, Robbins TW (2000) Probabilistic learning and reversal deficits in patients with Parkinson's disease or frontal or temporal lobe lesions: possible adverse effects of dopaminergic medication. Neuropsychologia 38:596-612.

Szabo J, Cowan WM (1984) A stereotaxic atlas of the brain of the cynomolgus monkey (Macaca fascicularis). J Comp Neurol 222:265-300.

Yahr MD, Duvoisin RC, Hoehn MM, Schear MJ, Barrett RE (1968) L-Dopa: its clinical effects in parkinsonism. Trans Am Neurol Ass 93:56-63. 